\title{
Trastuzumab/Vinorelbine Regimen
}

National Cancer Institute

\section{Source}

National Cancer Institute. Trastuzumab/Vinorelbine Regimen. NCI Thesaurus. Code C138053.

A chemotherapy regimen consisting of vinorelbine and trastuzumab, used as a treatment for recurrent and metastatic HER-2/neu-positive breast cancer. 